\title{
SOLITARY WAVES IN POISEUILLE FLOW OF A ROTATING FLUID
}

\author{
BY \\ CHIA-SHUN YIH \\ The University of Michigan, Ann Arbor, Michigan
}

Summary. Solutions are obtained for various modes of solitary waves in a strongly rotating fluid in Poiseuille flow. The nonlinear Bragg-Hawthorne equation is the basis of the theory, and the method of solution can be carried to any order of approximation. There are two sequences of modes, each infinite in number. One consists of modes travelling with the flow, called $\mathrm{P}$ modes, and the other consists of modes travelling against it, called $\mathrm{N}$ modes. Generally, the displacements of streamlines from their asymptotic positions are in opposite directions for two corresponding modes of the two sequences. In particular, the streamlines of the first mode travelling with the flow are displaced radially outward from their asymptotic positions, and those of the first mode travelling against the flow are displaced radially inward. In other words, the first $\mathrm{P}$ mode is a bulging wave, whereas the first $\mathrm{N}$ mode is a pinching or necking one.

For the interaction of solitary waves going in the same direction the Kortewegde Vries equation is derived. The beautiful solution of this equation by Gardner, Greene, Kruskal, and Miura (1967) can then be relied upon to provide the solution for the interaction problem. The $\mathrm{KdV}$ equation has already been formally derived by Leibovich (1969), who cited its solution of the $\operatorname{sech}^{2}$ form, but did not give the radial dependence of the stream function, so that the coefficients of his $\mathrm{KdV}$ equation have not been determined. Nor did he perceive the difference between the flow pattern for a $\mathrm{P}$ mode from that of the corresponding $\mathrm{N}$ mode.

1. Introduction. This paper is a study of inertial solitary waves in a strongly rotating fluid with a Poiseuille flow in the longitudinal direction and their interaction. The stated problem has already been investigated by Leibovich [4], who gave a mathematical scheme for deriving the Korteweg-de Vries equation, which, he noted, has a solution of the $\operatorname{sech}^{2}$ type, representing a solitary wave. But he did not determine the $r$-dependence of his stream function and therefore the coefficients of his $\mathrm{KdV}$ equation, and hence the parameters appearing in his $\operatorname{sech}^{2}$ solution are left undetermined. Here we treat the problem of one solitary wave as a steady-flow problem 
and arrive at its solution in a systematic way that can be continued to any order of approximation. This solution shows that a solitary wave travelling with the Poiseuille flow and another travelling against it have different flow patterns, even if the modal indices of the two waves are the same. This interesting feature seems to have escaped previous notice. The KdV equation, with definite coefficients, is then derived, and in the course of the derivation the concept of local long-wave velocity (including a nonlinear part) is introduced and that velocity (different from the velocity of a solitary wave, which is an "organized" wave) is determined.

2. Formulation of the problem of a solitary wave. We wish to inquire whether solitary waves are possible in a rotating inviscid fluid of constant density and, if so, to obtain a solution for their properties. For this purpose it is obviously necessary to have an equation governing the flow of rotating fluids. Such an equation has been provided by Bragg and Hawthorne [1], and for the sake of completeness and readability we shall reproduce briefly the derivation of their equation.

Since one can always move with any solitary wave, it is always possible to reduce the flow caused by an advancing solitary wave to a steady flow. Adopting cylindrical coordinates $(r, \theta, z)$, where $z$ is measured along the axis of symmetry and $r$ radially from it and $\theta$ is the azimuthal angle, and using $(u, v, w)$ for velocity components in the directions, respectively, of increasing $(r, \theta, z)$, the equations of motion for an inviscid fluid of constant density are, for axisymmetric flows,

$$
\begin{gathered}
\frac{D u}{D t}-\frac{v^{2}}{r}=-\frac{\partial}{\partial r}\left(\frac{p}{\rho}+\Omega\right), \\
\frac{D v}{D t}+\frac{u v}{r}=0 \\
\frac{D w}{D t}=-\frac{\partial}{\partial z}\left(\frac{p}{\rho}+\Omega\right),
\end{gathered}
$$

in which

$$
\frac{D}{D t}=u \frac{\partial}{\partial r}+w \frac{\partial}{\partial z},
$$

$p$ is the pressure, $\rho$ the density (assumed constant), and $\Omega$ the body-force potential. The equation of continuity is

$$
\frac{\partial(r u)}{\partial r}+\frac{\partial(r w)}{\partial z}=0,
$$

which allows us to use the Stokes stream function $\psi$, in terms of which

$$
w=\frac{1}{r} \psi_{r}, \quad u=-\frac{1}{r} \psi_{z},
$$

where subscripts indicate partial differentiation.

Equation (2) can be written as

$$
\frac{D}{D t}(v r)=0,
$$

which for steady flows means

$$
(v r)^{2}=f(\psi) .
$$


The function $f(\psi)$ in Eq. (7) is to be determined from the upstream condition in any particular problem. Then the other two equations of motion can be written as

$$
\begin{aligned}
& w\left(u_{z}-w_{r}\right)-\frac{1}{r^{2}} \Gamma \Gamma_{r}=-H_{r}, \\
& u\left(w_{r}-u_{z}\right)-\frac{1}{r^{2}} \Gamma \Gamma_{z}=-H_{z},
\end{aligned}
$$

where

$$
H=\frac{p}{\rho}+\frac{u^{2}+v^{2}+w^{2}}{2}+\Omega
$$

is the Bernoulli quantity, and $\Gamma=r v$. Cross differentiation of Eqs. (8) and (9) and use of Eq. (4) give

$$
\frac{D}{D t} \frac{\zeta}{r}+r^{-4} \frac{\partial}{\partial z} f(\psi)=0
$$

where $\zeta$ is the azimuthal component of the vorticity defined by

$$
\zeta=w_{r}-u_{z}=\frac{1}{r}\left(\psi_{r r}-\frac{1}{r} \psi_{r}+\psi_{z z}\right) .
$$

Equation (11) can be written as

$$
\frac{D}{D t} \frac{\zeta}{r}-u r^{-3} f^{\prime}(\psi)=\frac{D}{D t}\left(\frac{\zeta}{r}+\frac{f^{\prime}(\psi)}{2 r^{2}}\right)=0,
$$

which gives rise to the Bragg-Hawthorne equation

$$
\psi_{r r}-\frac{1}{r} \psi_{r}+\psi_{z z}+\frac{1}{2} f^{\prime}(\psi)=r^{2} h(\psi) .
$$

This equation is for steady flows only. But since the flow caused by a propagating solitary wave can be reduced to a steady flow by adopting a frame of reference moving with it, it is useful to the task at hand. The function $h$ is determined far upstream.

Consider stationary waves created by a fluid rotating with angular velocity $\omega$ and moving with constant velocity $-c$ past an obstacle at rest. Then far upstream, the velocity distribution is given by (if $c>$ linear long-wave velocity)

$$
u=0, \quad v=\omega r, \quad w=-c .
$$

(In this regard one notes that inertial waves due to rotation are like gravity waves in that the wave velocity increases with the wavelength, with the consequence that the group velocity is less than the phase velocity. This fact ensures that the waves created by any obstacle will be behind it.) Far upstream, then,

$$
\psi=-\frac{c r^{2}}{2}, \quad(v r)^{2}=\omega^{2} r^{4}=\frac{4 \omega^{2}}{c^{2}} \psi^{2}, \quad h(\psi)=-\frac{2 \omega^{2}}{c^{2}},
$$

and Eq. (12) becomes

$$
\psi_{r r}-\frac{1}{r} \psi_{r}+\psi_{z z}+\frac{4 \omega^{2}}{c^{2}} \psi=-\frac{2 \omega^{2}}{c} r^{2}
$$


This equation is exactly linear. It is to be solved with the boundary conditions

$$
w=\text { finite at } r=0 \quad \text { and } \quad \psi=-\frac{c b^{2}}{2} \text { at } r=b,
$$

where $b$ is the radius of the circular cylinder containing the fluid. Let

$$
\psi=-\frac{c}{2} r^{2}+r \phi
$$

Then Eq. (14) becomes

$$
\phi_{r r}+\frac{1}{r} \phi_{r}-\frac{1}{r^{2}} \phi+\phi_{z z}+\frac{4 \omega^{2}}{c^{2}} \phi=0
$$

which has the solution [5]

$$
\phi=A \exp (i k z) J_{1}(\lambda r / b),
$$

where

$$
\lambda=\left(\frac{4 \omega^{2}}{c^{2}}-k^{2}\right)^{1 / 2} b .
$$

To satisfy the boundary conditions on $\phi$ corresponding to Eq. (15), $\lambda$ must be any of the infinite number of denumerable numbers $\lambda_{n}$ satisfying

$$
J_{1}\left(\lambda_{n}\right)=0
$$

where $n$ is a mode index. The value of $c$ when $\lambda=\lambda_{n}$ will be denoted by $c_{n}$ so that

$$
\left(\frac{4 \omega^{2}}{c_{n}^{2}}-k^{2}\right)^{1 / 2} b=\lambda_{n},
$$

which is the dispersion equation for the waves. Clearly, for any mode, $c_{n}^{2}$ decreases as $k^{2}$ increases, so that the waves, called inertial waves, are like gravity waves in this regard, as noted before. The remarkable thing about Eq. (19) is that it holds for any amplitude of the waves, since Eq. (17) is exactly linear.

Now let $k^{2}$ decrease to zero. The speed of very long waves is given for any mode by

$$
\left(\frac{2 \omega b}{c_{n}}\right)^{2}=\lambda_{n}^{2}
$$

Suppose $c_{n}$ decreases beyond the limit given by Eq. (20). Then to satisfy Eq. (19) $k^{2}$ will have to be negative, indicating an exponential variation of $\phi$ in the $z$-direction. Then $\phi$ will increase exponentially in the positive or negative direction, which is not acceptable. For the wave motion to attentuate exponentially in both $z$-directions, one has to have a solitary wave which requires a nonlinearity in the governing differential equation, since, as we have just seen, the linear one will not do.

The nonlinearity can be provided by a variation in $v$ from that given by $\omega r$. But solid-body rotation is the one that can be realized most easily in the laboratory 
and should be preserved. That leaves the nonlinearity to be provided only by a shear flow. The most easily realized shear flow through a pipe is the Poiseuille flow

$$
w_{0}=W\left(1-\frac{r^{2}}{b^{2}}\right) .
$$

We note that both the solid-body rotation and the Poiseuille flow are realizable only if the fluid is viscous, and yet Eq. (12) is for an inviscid fluid. The apparent contradiction is resolved in the usual way. The Reynolds number based on $W$ is supposed to be high, so that the no-slip condition at $r=b$ as the solitary wave passes by can be satisfied by allowing a thin boundary layer at the solid boundary, in which viscous effects are fully taken into account. The core of the flow is governed by Eq. (12), for an inviscid fluid.

Let the solitary wave travel with velocity $c$ in the positive $z$-direction but allow $c$ to be positive or negative. If $c$ is positive, the wave travels with the flow. Then the velocity of the basic flow relative to the frame of reference moving with the wave is

$$
-c+w_{0}=-c+W-W(r / b)^{2},
$$

which has the Stokes stream function

$$
\Psi=-\frac{A}{2} r^{2}-\frac{B}{4} r^{4},
$$

with

$$
A=c-W, \quad B=W / b^{2} .
$$

Note that, since there is a longitudinal velocity, the value of $c$ for long waves is no longer given by Eq. (20) for any modal index $n$.

To determine $f(\psi)$ and $h(\psi)$ in Eq. (12), we consider the flow far upstream, where $\psi$ is equal to $\Psi$. Solving Eq. (23) for $r^{2}$, we have

$$
\begin{aligned}
r^{2} & =-(A / B)\left[1-\left(1-4 B \psi A^{-2}\right)^{1 / 2}\right] \\
& =-(2 / A)\left(\psi+B A^{-2} \psi^{2}+2 B^{2} A^{-4} \psi^{3}+\cdots\right),
\end{aligned}
$$

where $\psi$ has been written for $\Psi$. On the other hand,

$$
(v r)^{2}=\omega^{2} r^{4}=f(\psi) \text {. }
$$

Equations (25) and (26) give

$$
f(\psi)=\left(4 \omega^{2} / A^{2}\right)\left(\psi^{2}+2 B A^{-2} \psi^{3}+5 B^{2} A^{-4} \psi^{4}+\cdots\right)
$$

and

$$
\frac{1}{2} f^{\prime}(\psi)=\left(4 \omega^{2} / A^{2}\right)\left(\psi+3 B A^{-2} \psi^{2}+10 B^{2} A^{-4} \psi^{3}+\cdots\right) .
$$

As to $h(\psi)$, from the condition far upstream we have

$$
h(\psi)=-2 B+f^{\prime}(\psi) /\left(2 r^{2}\right),
$$

which, on account of Eq. (25), becomes

$$
h(\psi)=-2 B-\left(2 \omega^{2} / A\right)\left(1+2 B A^{-2} \psi+6 B^{2} A^{-4} \psi^{2}+\cdots\right) .
$$


We shall use the substitutions

$$
\psi=\omega b^{3} \hat{\psi}, \quad r=b \hat{r}, \quad z=b \hat{z}, \quad \alpha=\frac{\omega b}{A}, \quad \beta=\frac{B b}{\omega}=\frac{W}{\omega b}
$$

and assume $\beta \ll 1$. Then using Eqs. (30), substituting Eqs. (27) and (29) into Eq. (12), and dropping the circumflex signs, we have

$$
\psi_{r r}-\frac{1}{r} \psi_{r}+\psi_{z z}+4 \alpha^{2}\left(\psi+3 \alpha^{2} \beta \psi^{2}\right)=-2 r^{2}\left(\beta+\alpha+2 \alpha^{3} \beta \psi\right),
$$

from which terms of order $O\left(\beta^{2}\right)$ have been dropped.

We now take

$$
\psi=\Psi+r \phi, \quad \Psi=-\left(r^{2} / 2 \alpha\right)-\left(\beta r^{4} / 4\right) .
$$

Noting that $\Psi$ by itself satisfies Eq. (31) to the order $\beta$, we obtain from Eq. (31)

$$
\phi_{r r}+\frac{1}{r} \phi_{r}-\frac{1}{r^{2}} \phi+\phi_{z z}+4 \alpha^{2} \phi+24 \alpha^{4} \beta \Psi \phi+12 \alpha^{4} \beta r \phi^{2}=-4 \alpha^{3} \beta r^{2} \phi ;
$$

or, neglecting the term containing $\beta$ in the expression for $\Psi$ in Eq. (32),

$$
\phi_{r r}+\frac{1}{r} \phi_{r}-\frac{1}{r^{2}} \phi+4 \alpha^{2} \phi=8 \alpha^{3} \beta r^{2} \phi-12 \alpha^{4} \beta r \phi^{2}-\phi_{z z} \text {. }
$$

We now define the operator

$$
L_{1}=\frac{\partial^{2}}{\partial r^{2}}+\frac{1}{r} \frac{\partial}{\partial r}-\frac{1}{r^{2}}+\lambda_{n}^{2},
$$

where $\lambda_{n}$ satisfies Eq. (18). Since $\alpha^{2}$ must be a function of $\beta$ and the dimensionless amplitude $a$ (positive) of the basic component of the wave, we write, in view of Eq. (19),

$$
4 \alpha^{2}=\lambda_{n}^{2}+\beta G_{1}+\beta a G_{2},
$$

where $G_{1}$ and $G_{2}$ will be determined in the course of the calculation. Since the subscript $n$ in Eq. (35) is a mode index, logically $\alpha, G_{1}$, and $G_{2}$ should also have that index. But that would be tedious, and there is scant chance for misunderstanding by not adding that index.

Since we have decided to ignore quantities of order $O\left(\beta^{2}\right)$, in all terms of Eq. (34) where $\beta$ appears explicitly $\lambda_{n} / 2$ can be substituted for $\alpha$. Then

$$
L_{1} \phi=\lambda_{n}^{3} \beta r^{2} \phi-\frac{3}{4} \lambda_{n}^{4} \beta r \phi^{2}-\phi_{z z}-\left(\beta G_{1}+\beta a G_{2}\right) \phi,
$$

which is, finally, the equation on which the calculation will start. The boundary conditions are now

$$
\frac{1}{r}(r \phi)_{r} \text { is finite at } r=0, \quad \phi=0 \quad \text { at } r=1 .
$$

3. Solution and results for a solitary wave. We expand $\phi$ in a power series in $\beta$ and $a$, and, anticipating the $z$-dependence of $\phi$, we write

$$
\phi=S^{2}\left(a \phi_{0}+\beta a \phi_{1}+\beta a^{2} \phi_{2}+\cdots\right),
$$


where

$$
S=\operatorname{sech} \gamma z \text {. }
$$

Since the amplitude $a$ is fixed once and for all, $\phi_{1}, \phi_{2}$, etc., are made orthogonal to $\phi_{0}$. For the $n$th mode, the first approximation gives

$$
L_{1} \phi_{0}=0 \text {, }
$$

the solution of which is

$$
\phi_{0}=J_{1}\left(\lambda_{n} r\right) .
$$

That is indeed why we have chosen the first term in the expansion (35) for $4 \alpha^{2}$. Clearly Eq. (39) satisfies the boundary conditions (37).

For the next approximation we have

$$
L_{1} \phi_{1}=\lambda_{n}^{3} r^{2} \phi_{0}-G_{1} \phi_{0}=\left(\lambda_{n}^{3} r^{2}-G_{1}\right) J_{1}\left(\lambda_{n} r\right) .
$$

We now have to calculate the basic Fourier component containing $J_{1}\left(\lambda_{n} r\right)$ of the function $r^{2} J_{1}\left(\lambda_{n} r\right)$. This seems at first sight to require numerical integration. But we can indeed obtain what is needed by solving Eq. (40) to satisfy Eq. (36) and thereby obtain $G_{1}$ and $\phi_{1}$ in one step. The solution of $L_{1} \phi_{1}=q(r)$ can always be obtained if $q(r)$ is an even power of $r$ times $J_{1}\left(\lambda_{n} r\right)$. Yih's paper [6] on solutions of the hyper-Bessel equation contains material related to this point. For Eq. (40), it can be verified that

$$
\begin{gathered}
L_{1}\left[-\frac{1}{6 \lambda_{n}} r^{3} J_{0}\left(\lambda_{n} r\right)+\frac{1}{3 \lambda_{n}^{2}} r^{2} J_{1}\left(\lambda_{n} r\right)\right]=r^{2} J_{1}\left(\lambda_{n} r\right), \\
L_{1}\left[-\frac{1}{2 \lambda_{n}} r J_{0}\left(\lambda_{n} r\right)\right]=J_{1}\left(\lambda_{n} r\right) .
\end{gathered}
$$

Thus the solution of Eq. (40) is, with $\lambda_{n} r$ as the argument of the Bessel functions,

$$
\phi_{1}=\frac{1}{6}\left(-\lambda_{n}^{2} r^{3} J_{0}+2 \lambda_{n} r^{2} J_{1}+\frac{3 G_{1}}{\lambda_{n}} r J_{0}\right)+C_{1} \phi_{0},
$$

which satisfies (37) if

$$
G_{1}=\frac{1}{3} \lambda_{n}^{3}
$$

The $C_{1}$ in Eq. (43) is determined to make $\phi_{1}$ orthogonal to $\phi_{0}$ or

$$
\int_{0}^{1} r \phi_{0} \phi_{1} d r=0 \text {. }
$$

A formula useful for extending the calculation to higher orders of $\beta$ is

$$
L_{1}\left[-\frac{1}{10 \lambda} r^{5} J_{0}+\frac{3}{10 \lambda^{2}} r^{4} J_{1}+\frac{6}{5 \lambda^{3}} r^{3} J_{0}-\frac{12}{5 \lambda^{4}} r^{2} J_{1}\right]=r^{4} J_{1},
$$

and the other formulas needed can be discovered without difficulty.

For the next approximation we have

$$
\beta a^{2} L_{1} \phi_{2}=-\frac{3 \beta a^{2}}{4} S^{2} \lambda_{n}^{4} r \phi_{0}^{2}-4 \gamma^{2} a \phi_{0}+6 \gamma^{2} S^{2} a \phi_{0}-\beta a^{2} G_{2} \phi_{0} .
$$


The second and third terms on the right-hand side of Eq. (46) arise from $\phi_{z z}$. In order to proceed further, we need the basic Fourier component containing $J_{1}\left(\lambda_{n} r\right)$ in $r \phi_{0}^{2}$. Let that component be denoted by

$$
A_{n} J_{1}\left(\lambda_{n} r\right) \text {. }
$$

Then the solubility condition, i.e., that the right-hand side of Eq. (46) be orthogonal to $\phi_{0}$, requires that

$$
6 \gamma^{2}=\frac{3 \beta a}{4} \lambda_{n}^{4} A_{n} \quad \text { or } \quad \gamma^{2}=\frac{\beta a}{8} \lambda_{n}^{4} A_{n}
$$

and

$$
-4 \gamma^{2}=\beta a G_{2} \quad \text { or } \quad G_{2}=-\frac{4 \gamma^{2}}{\beta a}=-\frac{\lambda_{n}^{4}}{2} A_{n} .
$$

For the solution of $\phi_{2}$, we expand $r \phi_{0}^{2}$ in the series of eigenfunctions $J_{1}\left(\lambda_{m} r\right)$ :

$$
r \phi_{0}^{2}=\sum_{m=1}^{\infty} A_{m} J_{1}\left(\lambda_{m} r\right)
$$

The solution of Eq. (46) is then

$$
\phi_{2}=-\frac{3}{4} S^{2} \lambda_{n}^{4} \sum_{m}^{\prime} \frac{A_{m}}{\lambda_{n}^{2}-\lambda_{m}^{2}} J_{1}\left(\lambda_{m} r\right)
$$

in which the summation is over all integral values of $m$ from 1 onward except $n$. The exception is indicated by the prime on the summation sign. Thus $\phi_{2}$ is orthogonal to $\phi_{0}$.

The $A_{n}$ in Eq. (48) is evidently important. Unfortunately it has to be obtained numerically. For the first mode I have done some numerical integration by Simpson's rule, using the data given for $J_{1}(x)$ in the book by Jahnke and Emde [3, p. 157] and taking steps 0.1 for $x$ (except for the end interval) up to $x=\lambda_{1}=3.83$. The result is

$$
A_{1}=0.4368 \text {. }
$$

This is obtained from

$$
A_{1}=D^{-1} \int_{0}^{1} r^{2}\left[J_{1}\left(\lambda_{1} r\right)\right]^{3} d r=\left(D \lambda_{1}^{3}\right)^{-1} \int_{0}^{\lambda_{1}} x^{2}\left[J_{1}(x)\right]^{3} d x,
$$

where

$$
D=\int_{0}^{1} r\left[J_{1}\left(\lambda_{1} r\right)\right]^{2} d r=\frac{1}{2}\left[J_{0}\left(\lambda_{1}\right)\right]^{2} .
$$

To obtain $A_{n}$ for $n=2,3$, etc., simply change $\lambda_{1}$ to $\lambda_{n}$ in these formulas.

Equations (35), (44), and (49) give $\alpha^{2}$, which contains $c$. Equation (48) gives the wavelength (proportional to $\gamma^{-1}$ ) of the solitary wave, and Eqs. (38), (39), (43), and (51) give the eigenfunction for $\phi$. The solution can be carried to any order of approximation.

But for each $\alpha^{2}$ there are two values of $\alpha$, one positive and one negative, and this fact turns out to be far from trivial. For positive $\alpha$ ( $P$ modes), the wave is travelling 
with the Poiseuille flow, and for negative $\alpha$ ( $N$ modes), the wave is travelling against it. When we substituted $\lambda_{n} / 2$ for $\alpha$ on the right-hand side of Eq. (34) to get Eq. (36), we were focusing on the $P$ modes. For the $N$ modes, instead of Eq. (36) we obtain, upon substituting $-\lambda_{n} / 2$ for $\alpha$ on the right-hand side of Eq. (34),

$$
L_{1} \phi=-\lambda_{n}^{3} \beta r^{2} \phi-\frac{3}{4} \lambda_{n}^{4} \beta r \phi^{2}-\phi_{z z}-\left(\beta G_{1}+\beta a G_{2}\right) .
$$

Proceeding as before, we find that Eqs. (39), (49), and (51) still stand but that $G_{1}$ and $\phi_{1}$ are multiplied by -1 :

$$
G_{1}=-\frac{1}{3} \lambda_{n}^{3}, \quad \phi_{1 N}=-\phi_{1 P},
$$

where $\phi_{1 P}$ is just the $\phi_{1}$ given by Eq. (43).

Some interesting conclusions can now be drawn from the results. But we first clarify the nomenclature. We have been using $n$ as a mode index and calling the mode for $n=1$ the first mode. Then we find for each mode there is a $P$ mode and an $N$ mode. This need not create confusion. We can, and shall henceforth, call the modes the first $P$ mode, the $n$th $P$ mode, the first $N$ mode, and the $n$th $N$ mode, for instance.

From Eq. (35) and the definitions of $\alpha$ and $A$ given by Eqs. (30) and (24), using the $G_{1}$ given by Eq. (44) for the $P$ modes and by Eq. (54) for the $N$ modes, and the $G_{2}$ given by Eq. (49), we obtain

$$
c=\frac{2 W}{3} \pm\left(\frac{2 \omega b}{\lambda_{n}}+\frac{\lambda_{n} A_{n} a W}{2}\right)
$$

where the positive sign is for the $P$ mode and the negative sign for the $N$ modes.

An interesting feature of the solution concerns the flow patterns for the $P$ and $N$ modes. The equation for any streamline, on which $\psi$ is constant, is given by Eq. (32). If the amplitude is zero, the streamlines are straight, and their radial positions $r_{0}$ are given by

$$
\psi=\Psi=-(2 \alpha)^{-1} r_{0}^{2}-(\beta / 4) r_{0}^{4} .
$$

Because of the wave motion, the $r$ of any streamline will be a function of $z$, and we have, for the same value of $\psi$,

$$
r=r_{0}+\Delta r
$$

where $\Delta r$ is given by

$$
\left(\alpha^{-1} r_{0}+\beta r_{0}^{3}\right) \Delta r=r_{0} \phi
$$

the predominant part of which is

$$
\Delta r=\alpha a J_{1}\left(\lambda_{n} r_{0}\right) .
$$

In general, the displacements of the streamlines from their asymptotic positions are, for the $P$ modes, opposite for those for the $N$ modes. In particular, when $n=1$, the function $J_{1}\left(\lambda_{n} r\right)$ is positive in $0<r<1$, so that $\Delta r$ and $\alpha$ agree in sign, since Eq. (58) holds predominantly. In other words, when $n=1$, the solitary wave that 
travels with the Poiseuille flow is a bulging one, whereas that which travels against the flow is a pinching or necking one.

We could, of course, always let the wave propagate in the direction of increasing $z$, but for the $N$ modes we let the Poiseuille flow be directed in the opposite direction, i.e., let $\alpha$ be positive but $\beta$ be negative. If we adopt this point of view, the demand that $\gamma^{2}$, which was calculated from Eq. (48), be positive requires that the right-hand sides of Eqs. (39), (48), and (58) all acquire a minus sign. The same result is therefore obtained for the $N$ modes.

4. The Korteweg-de Vries equation. To study the interaction of solitary waves, we need an evolution equation treating unsteady long waves in the flow considered. Such an equation is the Korteweg-de Vries or KdV equation, which we now proceed to derive for waves of the same mode or $r$-structure. This will govern long waves going in the same direction. As mentioned before, Leibovich [4] obtained the KdV equation for the problem under treatment. But he did not give the $r$-dependence of his eigenfunction for the stream function, and hence the coefficients of his $\mathrm{KdV}$ equation have not been determined. Here we shall derive the $\mathrm{KdV}$ equation with the knowledge of the $r$-dependence of the eigenfunction and obtain that equation with definite coefficients. In the course of the derivation, a local nonlinear long-wave velocity will be defined and determined. The use of this local wave velocity clearly points out the interplay of nonlinear and dispersive effects in wave motion.

One starts from the Euler equations (1), (2), and (3), except that now the variables are dependent on $t$ and

$$
\frac{D}{D t}=\frac{\partial}{\partial t}+u \frac{\partial}{\partial r}+w \frac{\partial}{\partial z} .
$$

First Eq. (2) still gives Eq. (6), and Eq. (5) still stands, though Eq. (7) is no longer true. Let the dimensionless variables (with circumflex signs) in Eqs. (30) be again adopted. The first equation in (30) implies

$$
u=\omega b \hat{u}, \quad v=\omega b \hat{v}, \quad w=\omega b \hat{w} .
$$

In addition, let

$$
\hat{t}=\omega t, \quad \frac{p}{\rho}+\Omega=(\omega b)^{2} \chi .
$$

Then, substituting Eqs. (30), (60), and (61) into Eqs. (1) and (3) and dropping the circumflexes afterward, we have

$$
\begin{gathered}
\frac{D u}{D t}-\frac{v^{2}}{r}=-\chi_{r}, \\
\frac{D w}{D t}=-\chi_{z} .
\end{gathered}
$$

We still have

$$
\Gamma=r v
$$

but it is now dimensionless, and Eq. (6) can be written as

$$
\frac{D \Gamma}{D t}=0
$$


In Eqs. (62), (63), and (64), $D / D t$ is still given by Eq. (59), except that now all variables in it are dimensionless.

We now let

$$
w=w_{0}+w^{\prime}
$$

where $w_{0}$ is now dimensionless and given by

$$
w_{0}=\beta\left(1-r^{2}\right) \text {. }
$$

Substituting Eq. (65) into Eqs. (62) and (63) and eliminating $\chi$ by cross differentiation, we have

$$
\left(\frac{\zeta}{r}\right)_{t}+u\left(\frac{\zeta}{r}\right)_{r}+\left(w_{0}+w^{\prime}\right)\left(\frac{\zeta}{r}\right)_{z}=-\frac{2}{r^{4}} \Gamma \Gamma_{z}
$$

Let

$$
\zeta=\bar{\zeta}+\zeta^{\prime}, \quad \bar{\zeta}=-2 \beta r
$$

where $\bar{\zeta}$ arises from $w_{0}$ or $\Psi$ in

$$
\psi=\Psi+r \phi
$$

from which we have

$$
u=-\phi_{z}, \quad w^{\prime}=\frac{1}{r}(r \phi)_{r} .
$$

It is clear that $\bar{\zeta}$ makes no contribution to the left-hand side of Eq. (67), which can therefore be written as

$$
\zeta_{t}^{\prime}+u \zeta_{r}^{\prime}-\frac{u}{r} \zeta^{\prime}+\left[\beta\left(1-r^{2}\right)+w^{\prime}\right] \zeta_{z}^{\prime}=-\frac{2}{r^{3}} \Gamma \Gamma_{z}^{\prime},
$$

where

$$
\zeta^{\prime}=\phi_{r r}+\frac{1}{r} \phi_{r}-\frac{1}{r^{2}} \phi+\phi_{z z}
$$

On the other hand, Eq. (64) has the form

$$
\Gamma_{t}+u \Gamma_{r}+\left(w_{0}+w^{\prime}\right) \Gamma_{z}=0
$$

With

$$
\Gamma=r^{2}+\Gamma^{\prime}
$$

Eq. (70) becomes

$$
\Gamma_{t}^{\prime}+\beta \Gamma_{z}^{\prime}=-2 r u+\beta r^{2} \Gamma_{z}^{\prime}-\left(u \Gamma_{r}^{\prime}+w^{\prime} \Gamma_{z}^{\prime}\right) .
$$

We shall work with Eq. (69) first and shall evaluate $\zeta^{\prime}$ from Eq. (40) and the equation just before Eq. (39). This is permissible since the unsteady state under treatment deals with any local condition, and if one moves with the local long-wave velocity, one can reduce the local flow to a quasi-steady state. From Eq. (69) and the definition of $L_{1}$ following Eq. (34), we have

$$
\zeta^{\prime}=L_{1} \phi-\lambda_{n}^{2} \phi+\phi_{z z}
$$

We have shown in Sec. 3 that $L_{1} \phi_{0}=0$ and $L_{1} \phi_{1}$ is orthogonal to $\phi_{0}$. Indeed, anticipating Eq. (76), we see that $L_{1} \phi$ is orthogonal to $\phi_{0}$ or to $\phi$ if a term of 
$O\left(\beta^{2}\right)$ is neglected. Since eventually the mode $\phi_{0}+\beta \phi_{1}$ will be extracted, we can write, for the linear terms,

$$
\zeta^{\prime}=-\lambda_{n}^{2} \phi+\phi_{z z}
$$

For the left-hand side of Eq. (68), let

$$
\mathrm{I}=\zeta_{t}^{\prime}+\beta \zeta_{z}^{\prime}=\left(1-\frac{\beta}{c}\right)\left(-\lambda_{n}^{2} \phi_{t}+\phi_{t z z}\right)
$$

where $c$ is the long-wave velocity,

$$
\mathrm{II}=-\beta r^{2} \zeta_{z}^{\prime}=\beta \lambda_{n}^{2} r^{2} \phi_{z},
$$

with the term $-\beta r^{2} \phi_{z z z}$ neglected, and

$$
\mathrm{III}=u \zeta_{r}^{\prime}+w^{\prime} \zeta_{z}^{\prime}-\frac{u \zeta^{\prime}}{r}=-\phi_{z} \zeta_{r}^{\prime}+\phi_{r} \zeta_{z}^{\prime}+\frac{1}{r}\left(\phi \zeta_{z}^{\prime}+\phi_{z} \zeta^{\prime}\right)
$$

In calculating III, we must include the right-hand side of Eq. (40) to calculate $\zeta^{\prime}$ but shall neglect terms of $O\left(\beta^{2} a^{2}\right)$ and $O\left(\beta a^{3}\right)$. With $f(z, t)$ now replacing the $a$ in Eqs. (35) and (38), we have

$$
\phi=f(z, t)\left(\phi_{0}+\beta \phi_{1}\right)
$$

for the calculation of III; and, after some calculation with Eq. (72), the equation just before (39) and Eq. (40), we obtain

$$
\mathrm{III}=-\frac{2 \lambda_{n}^{2} \phi \phi_{z}}{r}-\frac{2 \beta \lambda_{n}^{3}}{3 r} \phi_{0}^{2} f f_{z},
$$

if terms of $O\left(\beta^{2}\right)$ are neglected. Note that the first term on the right-hand side of Eq. (40) does not contribute to the final result for III. The $f$ from Eq. (76) onward is not the $f$ in Eq. (7).

We now turn to Eq. (71). First, by using the linear dispersion equation

$$
c_{1}(\text { linear wave speed })=c_{0}\left(1-\frac{k^{2}}{2 \lambda_{n}^{2}}\right)
$$

obtained from the equation above Eq. (18), we have

$$
\Gamma_{t}^{\prime}=-c \Gamma_{z}^{\prime}-\frac{c}{2 \lambda_{n}^{2}} \Gamma_{z z z}^{\prime}
$$

then we can write Eq. (71) as

$$
\begin{aligned}
-\hat{c} \Gamma_{z}^{\prime} & =-(c-\beta) \Gamma_{z}^{\prime} \\
& =2 r \phi_{z}+\beta r^{2} \Gamma_{z}^{\prime}+\phi_{z} \Gamma_{r}^{\prime}-\frac{1}{r}(r \phi)_{r} \Gamma_{z}^{\prime}+\frac{c}{2 \lambda_{n}^{2}} \Gamma_{z z z}^{\prime} .
\end{aligned}
$$

In Eqs. (78) and (79)

$$
c=c_{0}+c^{\prime}, \quad \hat{c}=c-\beta,
$$


$c_{0}$ is the linear long-wave velocity, and $c^{\prime}$ is the local nonlinear part of the total long-wave velocity and is of order $O(\beta f)$. Equation (35) gives, in view of Eq. (24),

$$
\frac{4}{\left(c_{0}-\beta\right)^{2}}=\lambda_{n}^{2}+\frac{\beta}{3} \lambda_{n}^{3} \text {. }
$$

The first approximation of Eq. (79) is

$$
\Gamma^{\prime}=-\frac{2}{\hat{c}}\left(1-\frac{\beta r^{2}}{\hat{c}}\right) r \phi .
$$

A second iteration using Eq. (82) gives, neglecting higher-order terms in the last term in Eq. (79),

$$
\Gamma_{z}^{\prime}=-\frac{2 r}{\hat{c}} \phi_{z}+\frac{2 \beta}{\hat{c}^{2}} r^{3} \phi_{z}-\frac{4 \beta r^{2}}{\hat{c}^{3}} \phi \phi_{z}+\frac{r}{\hat{c} \lambda_{n}^{2}} \phi_{z z z} .
$$

Therefore,

$$
\Gamma^{\prime}=-\frac{2 r}{\hat{c}} \phi+\frac{2 \beta}{\hat{c}^{2}} r^{3} \phi-\frac{2 \beta r^{2}}{\hat{c}^{3}} \phi^{2}+\frac{r}{\hat{c} \lambda_{n}^{2}} \phi_{z z} .
$$

From Eqs. (82) and (83) we have, since $c_{0}-\beta$ is given by Eq. (81),

$$
\begin{aligned}
-\frac{2 \Gamma}{r^{3}} \Gamma_{z}^{\prime} & =-\frac{2\left(r^{2}+\Gamma^{\prime}\right)}{r^{3}} \Gamma_{z}^{\prime} \\
& =\frac{4}{\hat{c}} \phi_{z}-\frac{4 \beta}{\hat{c}^{2}} r^{2} \phi_{z}-\frac{8}{\hat{c}^{2} r} \phi \phi_{z}+3 \beta r \lambda_{n}^{3} \phi \phi_{z}-\frac{2}{\hat{c} \lambda_{n}^{2}} \phi_{z z z} .
\end{aligned}
$$

Using Eqs. (80) and (81) on the third term of the right-hand side of this equation and ignoring terms of order $O\left(\beta^{2}\right)$, we can write Eq. (85) as

$$
-\frac{2 \Gamma}{r^{3}} \Gamma_{z}^{\prime}=\frac{4}{\hat{c}} \phi_{z}-\beta \lambda_{n}^{2} r^{2} \phi_{z}-\frac{2 \lambda_{n}^{2}}{r} \phi \phi_{z}-\frac{2 \beta}{3 r} \lambda_{n}^{3} \phi \phi_{z}+3 \beta r \lambda_{n}^{3} \phi \phi_{z}-\frac{1}{\lambda_{n}} \phi_{z z z} .
$$

Now Eq. (68) can be written as

$$
\mathrm{I}+\mathrm{II}+\mathrm{III}=-\frac{2 \Gamma}{r^{3}} \Gamma_{z}^{\prime} .
$$

It is clear that III cancels the third and fourth terms on the right-hand side of Eq. (86), so that Eq. (87) becomes

$$
\left(1-\frac{\beta}{c}\right)\left(-\lambda_{n}^{2} \phi_{t}+\phi_{t z z}\right)=\frac{4}{\hat{c}} \phi_{z}-2 \beta \lambda_{n}^{2} r^{2} \phi_{z}+3 \beta r \lambda_{n}^{2} \phi \phi_{z}-\frac{1}{\lambda_{n}} \phi_{z z z} .
$$

With $\phi_{t z z}=-c \phi_{z z z}$ and Eq. (81), this can be written as

$$
\phi_{t}+\left(\frac{4}{\lambda_{n}^{2} \hat{c}}+\beta-2 \beta r^{2}\right) \phi_{z}+3 \beta r \lambda_{n} \phi \phi_{z}+\frac{1}{\lambda_{n}^{3}} \phi_{z z z}=0 .
$$

Extracting from Eq. (88) the terms containing $\phi$ as a factor and noting that, since the right-hand side of Eq. (40) is orthogonal to $\phi_{0}$, we may replace $r^{2} \phi_{0}$ by $\phi_{0} / 3$ when doing this extraction, we obtain

$$
f_{t}+\left(\frac{4}{\lambda_{n}^{2} \hat{c}}+\frac{\beta}{3}\right) f_{z}+3 \beta \lambda_{n} A_{n} f f_{z}+\frac{1}{\lambda_{n}^{3}} f_{z z z}=0,
$$

where $A_{n}$ is as defined in Eq. (47). 
We now calculate $c^{\prime}$, the nonlinear part of the long-wave velocity. Since $c_{0}$ is given by Eq. (81), we have, neglecting terms of $O\left(\beta^{2}\right)$,

$$
\frac{4}{\lambda_{n}^{2} \hat{c}}=\frac{\left(c_{0}-\beta\right)^{2}}{c_{0}-\beta+c^{\prime}}\left(1+\frac{\beta}{3} \lambda_{n}\right)=c_{0}-\frac{\beta}{3}-c^{\prime} .
$$

Then, since we are seeking the wave velocity for long waves, we can drop the last term in Eq. (89) and obtain

$$
-c_{0}-c^{\prime}+c_{0}-c^{\prime}+3 \beta \lambda_{n} A_{n} f=0
$$

or

$$
c^{\prime}=\frac{3 \beta}{2} \lambda_{n} A_{n} f .
$$

Substituting this into Eq. (89) and using Eq. (90), we have, finally,

$$
f_{t}+\left(c_{0}+\frac{3 \beta}{2} \lambda_{n} A_{n} f\right) f_{z}+\frac{1}{\lambda_{n}^{3}} f_{z z z}=0
$$

which is the $\mathrm{KdV}$ equation, for waves going in the direction of increasing $z$. For waves going in the direction of decreasing $z$, we first change the sign of the last term in Eq. (81), which is $\beta G_{1}$, and then solve Eq. (81) for a negative $c_{0}$. The result is then substituted into Eq. (92) to obtain the $\mathrm{KdV}$ equation for $N$ modes. The beautiful solution given by Gardner, Greene, Kruskal, and Miura [2] then provides the answer to the problem of interaction of solitary waves of the same mode and going in the same direction.

For a single solitary wave, it can readily be shown that Eq. (92) gives exactly the same results, including the wavenumber $\gamma$ and the wave speed, as those given in Sec. 3, now for the laboratory frame of coordinates. This is as it should be and is reassuring.

\section{REFERENCES}

[1] S. L. Bragg and W. R. Hawthorne, Some exact solutions of the flow through annular cascade actuator discs, J. Aeronaut. Sci. 17, 243-249 (1950)

[2] C. S. Gardner, J. M. Greene, M. D. Kruskal, and R. M. Miura, Method for solving the Korteweg-de Vries equation, Phys. Rev, Lett 19, 1095-1097 (1967)

[3] E. Jahnke and F. Emde, Table of Functions, Dover, New York, 1945

[4] S. Leibovich, Weakly nonlinear waves in rotating fluids, J. Fluid Mech. 42, 803-822 (1969)

[5] R. R. Long, Steady motion of a symmetric obstacle moving along the axis of a rotating fluid, J. Meteorology 10, 197 (1953)

[6] C.-S. Yih, Solutions of the hyper-Bessel equation, Quart. Appl. Math. XIII, 462-463 (1956) 